\title{
Eficacia en la atención hospitalaria de pacientes COVID-19 en el Hospital Ángeles Chihuahua
}

\author{
Efficiency in hospital care of COVID-19 patients \\ at Hospital Ángeles Chihuahua
}

\author{
Jesús Eduardo Romo Martínez, * Rodolfo Chávez Chávez, \\ Rogelio Reyes Soto, § Raúl Hernández Saldaña, "Ivonne Loera Estrada," \\ Sandra Rivas Cobos, ${ }^{* *}$ Giselle Duarte Duarte**
}

Citar como: Romo MJE, Chávez CR, Reyes SR, Hernández SR, Loera El, Rivas CS et al. Eficacia en la atención hospitalaria de pacientes COVID-19 en el Hospital Ángeles Chihuahua. Acta Med Grupo Angeles. 2021; 19 (3): 404-405. https://dx.doi.org/10.35366/101739

Respetable editor:

Hospital Ángeles Chihuahua cuenta con una capacidad instalada en capital humano, infraestructura y servicios suficientes para la atención de pacientes bajo protocolo SARS-CoV-2 en la presente pandemia por COVID-19, aunado al ejercicio profesional bajo el modelo de seguridad del paciente. Por lo que a través del presente ponemos a disposición de su consejo editorial la siguiente información explicita en el Sistema Nacional de Vigilancia Epidemiológica (SINAVE), con el fin de exponer de manera objetiva la eficacia del manejo hospitalario en dichos casos, que se ve reflejada en un manejo exitoso tras ratificar su mejoría y curación.

Desde abril 2020 y hasta mediados de junio 2021 se ha brindado atención a casi dos mil pacientes, tanto en manejo ambulatorio como hospitalario. De este último grupo, se calcula una eficacia en la gran mayoría (en $84.3 \%$ de un total de 842 pacientes).

Ahora bien, valdría la pena analizar qué factores pudieron interactuar en ese $15.7 \%$ restante y además dimensionar si pudieran relacionarse o no al proceso de atención.

Analizando de manera cuantitativai, la edad de los pacientes hospitalizados denota una diferencia de promedios contundente en quienes tuvieron un motivo de egreso por mejoría de 53.7 años versus 66.7 años que egresaron por defunción $(p \leq 0.001)$. Los días promedio para solicitar atención médica no mostraron una diferencia con significancia estadística ( $p=0.23$ ), observando poco más de seis días independientemente del desenlace. En cuanto al promedio de días de estancia hospitalaria, se evidenció 9.1 promedio de días estancia en los egresados por mejoría versus 14.5 promedio de días estancia en egresados por defunción $(p \leq 0.001)$.

Un análisis cualitativo $o^{\mathrm{ii}}$ nos dice que el antecedente de hipertensión o afección renal crónica condiciona a los pacientes a tener el doble de posibilidades de no alcanzar la eficacia deseable $(p \leq 0.001)$. En caso de tener una sobreestancia hospitalaria (mayor o igual a siete días), se observa un riesgo casi tres veces mayor de no alcanzar la eficacia $(p \leq 0.001)$; mientras que para situaciones de atención en área de cuidados intensivos con ventilación mecánica, condicionó hasta nueve veces más la probabilidad de tener un desenlace en mortalidad $(p \leq 0.001)$.

En resumidas cuentas, la atención hospitalaria de pacientes COVID-19 en Hospital Ángeles Chihuahua ha tenido una alta eficacia. Las condicionantes que parecieran eclipsar este desempeño han resultado, por lo general,

\footnotetext{
i Evaluación estadística mediante prueba t de Student.

ii Evaluación estadística mediante estimación odds ratio con $\chi^{2}$.
}

\footnotetext{
* Jefe de Unidad de Vigilancia Epidemiológica Hospitalaria.

₹ Director General.

§ Dirección Médica.

" Líder de estrategia COVID-19.

|| Dirección de Planeación y Calidad.

** Unidad de Vigilancia Epidemiológica Hospitalaria.
}

Hospital Ángeles Chihuahua. México.
Correspondencia:

Jesús Eduardo Romo Martínez

Correo electrónico:

epidemiologia@angeleschihuahua.com

www.medigraphic.com/actamedica

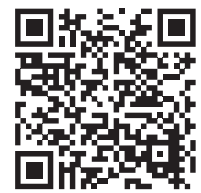


no atribuibles al proceso de atención por ser inherentes a las condiciones propias de los pacientes como la edad, enfermedades crónicas y complicaciones, sobreestancia hospitalaria que puede ser atribuible a interacción de estas comorbilidades con el SARS-CoV-2.
En el futuro, valdría la pena ejercer análisis más extensos, multicéntricos y con información más detallada para conocer la "otra cara de la moneda" en la pandemia por COVID-19... tener confianza en que los procesos de atención hoy por hoy se llevan de manera eficaz. 